\title{
Online Signature Verification: State of the art
}

\author{
I.M. El-Henawy1, M. Z. Rashad2, O. Nomir2, K. Ahmed2 \\ 1 Zagazig University, Computer Science Department \\ henawy2000@yahoo.com
}

2 Mansoura University, Computer Science Department

magdi_12003@yahoo.com, omnomir@yahoo.com, kareem_ahmed@hotmail.co.uk

\begin{abstract}
online or Dynamic signature verification (DSV) is one of the most acceptable, intuitive, fast and cost effective tool for user authentication. DSV uses some dynamics like speed, pressure, directions, stroke length and pen-ups/pen-downs to verify the signer's identity. The state of the art in DSV is presented in this paper. several approaches for DSV are compared and the most influential techniques in this field are highlighted. We concentrate on the relationship between the verification approach used (the nature of the classifier) and the type of features that are used to represent the signature.
\end{abstract}

\section{Literature Review}

The signing process can be described at two levels: at the high level, the signing method is recovered from long-term memory; parameters are then specified such as size, shape, timing; finally, at a peripheral level, commands are generated for the biophysical muscles. So, the signing process is believed to be a reflex action (ballistic action), rather than a deliberate action. Ballistic handwriting is characterized by a spurt of activity, without positional feedback, whereas deliberate handwriting is characterized by a conscious attempt to produce a visual pattern with the aid of positional feedback [25]. The problem arises here is that many signers can produce their signatures both ballistically and deliberately based on the nature and importance of the task. The process of Signature verification is a two-class problem where the input signature is classified as genuine or forged (i.e. belonging to an impostor). The simplest approach to compare two signatures is to compute correlations between the test signature values and the reference signature values. Such point-topoint comparison usually does not work well since there are a large number of variations between the two signatures and the correlation can be affected by the translation, rotation or scaling of the signature. The decision is made by comparing the similarity score between the input and the enrolled signatures with a predetermined threshold. A tablet device is used to capture dynamic information of a signature. Such dynamic information is a set of sequences of sampled points over time. Each sequence depicts an action performed during the signing process. Signatures from the same person may have different trajectory lengths due to local stretching, compression, omission or additional parts, and hence they are represented by feature vectors of differing lengths. Therefore, straight forward methods, such as the Euclidian distance or autocorrelation, are not very useful in calculation of the dissimilarity value between two signatures [6]. Such point-to-point comparison usually does not work because some portions of any two genuine signatures of the same person may vary significantly. To overcome the problem, non-linearly approaches such as Dynamic Time Warping (DTW) algorithm and Hidden Markov Models (HMM) are commonly used in aligning two signatures. Plamondon and Lorette [141] categorized the various signature verification methods into two types: (i) parametric (Feature)-based approach: In which, each time a signature is presented to the system, equal number of global features derived from the acquired signature trajectories are extracted then a distance measure (ex. Euclidian distance) is used to compare reference and test signatures, and (ii) functional-based approach: In which time sequences describing local properties of the signature are used for verification (e.g., position trajectory, velocity, acceleration, pressure). In the parametric approach, a complete feature vectors are extracted from the signatures and compared to obtain similarity measures. In this approach, once a set of features has been selected, only the values of these features are stored. Parametric approach may also called signature compression because it only needs to store a set of features' values which may be useful if, for example, the reference signature needs to be stored on a card. When a test signature is presented, only the features' values are extracted and compared, not the signature. The main research issues [26] on the parametric approach are: (i) How many global features are sufficient?, (ii) Do the signatures require some transformation before computing the features, for example resizing, rotating or smoothing?, (iii) How many sample signatures will be used in computing the reference signature?, (iv) Would the reference signatures be updated regularly? If yes, how often it will be updated?, (v) Should user-dependent set of features be used rather than the same features for all?, (vi) Should user-dependent threshold be used rather than the same for all?, (vii) How is the distance between a test signature and the reference signature is computed?. When the algorithm uses a large number of features, a single feature can unduly influence the decision through deviating far from the mean value, even if the other features have values close to their means for the genuine reference set. To solve this problem, it either must have a large threshold for the norm of the distances or use some criterion similar to the Majority Classifier (MC) [12]. The Majority Classifier (MC) accepts a test signature as a genuine one if the number of feature values which pass a predetermined test is larger than half the total number of tested features. Function-based approach treats the signing process as a set of one-dimensional discrete time functions and performs the matching process based on an elastic measure such as DTW $[7,9,14,15,17,18,31,36,44,45,51,54,67,71,72,79,81,84,85,86,93,96,98,99,101,104$, $106,112,122,124,126,129,140,142,143,146,163,167,200,221,222]$ or HMM $[2,5,21,29,30,32,58,61,73,128$, $131,132,135,149,155,157,168,175,187,192,203,207,216,218,223,224,225]$. DTW performs flexible matching of local features of two signatures. HMM performs stochastic matching of the two signatures using a sequence of probability distributions of the features along the signature. The major research issues [26] on the functional approach are: (i) How 
many local functions should be used?, (ii) How the comparison is achieved given that genuine signatures of an individual tend to have some variation amongst them?, (iii) If the signature is divided into segments, how would segmentation take place?, (iv) How would the distance between the test signature and the reference signatures be computed?. Hansheng Lei and Venu Govindaraju [1, 206] studied the consistency and discriminative power of the time-based functions, and reported that the simple features like $\mathrm{X}$-, $\mathrm{Y}$ - coordinates, the speed of writing and the angle with the X-axis are the most consistent. Function-based approaches can be extended into local and regional methods. Local methods directly match the time functions of different signatures by using elastic distance measures such as Dynamic Time Warping. Regional methods convert time functions to a sequence of vectors that describe the regional properties of the signature. The most popular regional approach is the HMM. Since the signature trajectory can be viewed as a function of time, a reliable dissimilarity comparison method must meet following criteria: (i) Difference of overall signal duration, (ii) Existence of non-linear distortions within signals, (iii) The length of a pen input trajectory can be different from that of stored genuine signature, (iv) Number of obtained strokes (pen-ups/pen-downs) of a pen input trajectory may be different from that of stored genuine signature.

\section{FEATURES-BASED APPROACHES}

Depending on the type of the capturing device, data such as $\mathrm{x}, \mathrm{y}$ coordinates, pen velocity, pen pressure and pen tilt are recorded. A fixed number of global features is extracted from these data and arranged in a feature vector which describes discriminative characteristics of the signature and stored as a template. Gupta and Joyce [162] studied the effect of using six global features on the performance of the verification system. The features studied are: total time, the total pen-up time, number of velocity sign changes in the $x$ - and $y$-direction, number of acceleration sign changes in the $x$ - and $y$ direction. There are many different distance metrics [26] that can be used to compute the distance between a test signature and the corresponding reference signature. The most common method to find the similarity between the input feature vector and the stored template is to use a classical distance measure such as Euclidean distance, Mahalanobis distance, Canberra Distance, Euclidean Distance, City Block distance and Hamming Distance. Some of the algorithms that used features-based approaches can be found in $[4,9,12,22,28,33,49,52,65,93,94,107,139,161,162,179$, $193,195,199,200,205,208,209,210,215]$. L. Lee et al. [12] discussed a number of techniques for feature selection from a set of 42 features and 49 normalized features. Kim et al. [33] studied 75 features and selected the best combination of features for each individual using an evaluation function called the Degree of Difficulty to Forge (DDF). Ketabdar et al. [49] selected the most discriminative global features by combining a modified Fisher ratio cost function and a near-optimal feature space search algorithm. Taherzadeh et al. [161] extracted 30 features from the online signature and then categorized them into four categories: Direction-related, Pressure-related, Dynamic-related and Shape-related. Carlos Vivaracho-Pascual et al. [205] proposed a DSV system that is new in both feature extraction and classification stages. The proposed approach is based on the use of size normalized signatures, which allows similarity estimation to be performed by an easy distance calculation between vectors. They used the fractional distance, instead of the more typical Euclidean one, so as to overcome the concentration phenomenon that appears when data are high dimensional. David Aristizábal et al. [208] proposed a DSV system that extracts 42 features from seven time-series functions by applying 6 nonlinear dynamic techniques to each series. Julio Romo and Rogelio Silva [209] proposed a DSV system based on creating Optimal Prototype Functions (OPF) of the discriminant features of the signatures. Chang and Hong-De [28] proposed a DSV system of Chinese signatures based on Bayesian neural networks.

\section{Dynamic Time Warping (Elastic Matching)}

After modeling each signature by a set of time based vectors, there must be a method to compare signatures based on their vector representations. Since there are variations among genuine signatures of the signer, the time based vectors representing these signatures may be of different lengths which makes it hard to use a naive element-wise comparison of signatures, such as Euclidian distance between two vectors. One of the simplest and successful approaches for online signature verification is DTW. DTW is used to measure similarity between two sequences that vary in time or speed. DTW finds the best nonlinear alignment of two vectors such that the overall distance between corresponding vector elements is minimized in least square sense. In DTW the input and reference signatures are compared by using dynamic programming (DP) matching algorithm which can manage the variability on the signature's length. DTW is used to find the best matching path, in terms of the least global cost, between the test signal and the stored template. The algorithm is a well-known and widely used for aligning vectors of different lengths. This approach is also called elastic matching or elastic distance because it is tolerant to local variations in the compared signatures. DTW finds an optimal match between two sequences that may contain stretched or compressed sections. The idea falls behind that we can predict missing information in the compared sequence, if we have long enough segments to match. DTW has been widely used in online signature verification systems to cope with variable length of signatures. The approach relies on minimization of a global cost function that contains local distances between the two signatures that are compared. Many researchers use DTW to obtain a point to point correspondence between two similar signatures. DTW is used to accommodate the timing but not the $X$ and $Y$ coordinates of the signature. By applying DTW to $X$ and $Y$ signals, it will distort the shape of signature. DTWbased techniques can be further divided into several sub-categories: Full Signal Warping[14, 15, 51, 72, 79, 81, 85, 176, 212], Extreme Points Warping[17, 44, 45], Shape contexts DTW [78, 83, 194], Continuous DTW[67, 71, 86, 99, 101, 114, 167, 214], Mahalanobis-based DTW[45, 106, 140, 158], PCA, NCA and MCA based DTW [18, 146, 226], Bidirectional backward-merging DTW[147], GA and PA-based DTW[121, 143]. 


\section{Hidden Markov Model (HMM)}

Hidden Markov Models (HMM) were introduced in the pattern recognition field as a robust method to model the variability of discrete time random signals where time or context information is available[19]. HMMs are widely used in classification of input patterns and they have a great ability to model stroke-based sequences. These models have found to be well suited for signature modeling since they are highly adaptable to personal variability[80]. The underlying assumption of the $\mathrm{HMM}$ is that the signal can be well characterized as a parametric random process, and that the parameters of the stochastic process can be estimated in a precise, well-define manner[20]. In HMM, the probability of moving from one state to another depends on both the transition probabilities and the previously visited states. The left-to-right topology is the most applicable in DSV systems since it is considered well suited for signature modeling. some of the techniques that used Ergodic topology can be found in [135, 168]. Juan J. Igarza et al.[105] compared between different topologies used for DSV and reported that the worst results were obtained using Ergodic or generalized models, in which transitions between all the states are allowed. Donato Impedovo and Giuseppe Pirlo[80] reported that the ring topology can be used to guarantee rotation invariance of the signatures. the ring topology is equivalent to left-to-right topology with a transition from the last state to the first state. An algorithm such as Viterbi algorithm can be combined with HMMs to find the most likely state sequence that generates the observed sequence. Other algorithms such as the Baum-Welch or the Segmental K-means can be used to update the parameters of the model to maximize the probability of the sequence generated by the model. Julian Fierrez-Aguilar et al. $[19,22]$ proposed applying HMM to 14 time sequences derived from dynamic functions. Ly-Van et al. [30, 149] proposed an HMM-based DSV approach, that fuses two complementary information levels issued from the same continuous HMM with a multivariate Gaussian mixture in each state. Yang et al. [37] extracted normalized angles along signature trajectory and model the generation of this sequence by HMMs. Dolfing et al. [38] proposed a DSV system in which each signature is modeled by a single HMM without any preprocessing. Rigoll and Kosmala [39] presented the first systematic comparison of on-line and off-line methods for signature verification using HMM on the same database. Ortega-Garcia et al. [43] proposed a function-based approach to online signature verification using an initial set of 8 time sequences. Kashi et al. [55, 87] proposed a DSV system using local and global features where Local features of inclination angle and difference between adjacent angles are used to build a left-to-right HMM model. Fierrez-Aguilar et al. [59] studied the two function-based approaches for DSV. These approaches are divided into local and regional depending on the features used for matching. The authors made some modifications to the local approach using DTW that was proposed by Jain et al. [24]. It was found that the DTW system outperforms the HMM system. It was shown that combining DTW and HMM and employing user-dependent thresholds performs significantly better than individual DTW or HMM systems. M. Shafiei and H. Rabiee [90] proposed a DSV system Using Variable Length Segmentation and HMM. Juan J. Igarza et al. [105] presented a research of various HMM based techniques for DSV. Different topologies are compared in order to obtain an optimized high performance signature verification system. DawTung Lin and Yu-Chia Lia [128] presented a DSV system using Hidden Semi-Markov Model (HSMM). Zhong-Hua Quan and Kun-Hong Liu [135] proposed an online signature verification system based on Ergodic HMM and a time delay Neural Networks. Muramatsu and Matsumoto [155] proposed a HMM-based approach for online signature verification using only pen position trajectories. Pascual-Gaspar and Cardenoso-Payo [175] proposed an online Signature Verification system based on HMM with user-dependent structure. Colin Sindle [131] presented a DSV system based on HMM that uses only the local features after some novel preprocessing steps. Enrique Argones et al. [168] proposed a novel approach for online signature verification based on building HMM user models by adapting an Ergodic Universal Background Model (UBM) with a fixed number of states. M. Martinez-Diaz et al. $[138,197]$ presented an HMM-based DSV system in which the number of states and Gaussian mixtures of the HMM are specifically set for each user. It was shown that when using the user-dependent structure, the system error rate is significantly decreased. Mitsuru Kondo et al. [60] proposed a DSV approach by performing nonlinear separation using Bayesian Markov Chain Monte Carlo (MCMC). Daigo Muramatsu et al. [46] used a similar approach to that proposed in [60] except that they used four distance measure. The fourth distance measure is the data length difference.

\section{Gaussian Mixture Models (GMM)}

Gaussian Mixture Models (GMM) are considered to be a stable classifier because small variations in the sampling of the training set will produce not a large change in the parameters of the trained classifier. Some of the techniques that used GMM are Jonas Richiardi and Andrzej Drygajlo [27], Martinez-Diaz et al. [23], Andreas Humm [63], JUGURTA MONTALVÃO [156], Wan and Liang [192], Jonas Richiardi et al. [198], Riccardo Corradin [220]. GMMs are well-known flexible modeling tools can approximate any probability density function. Richiardi et al. [27] was the first one who introduced and motivated the use of GMMs for DSV. JUGURTA MONTALVÃO [156] proposed another model for DSV based on GMM which is a degenerated version of an HMM with only one state. Andreas Humm et al. [63, 150, 151] proposed a novel multimodal user authentication system based on a Combined Handwriting and Speech Modalities (CHASM). Mandeep Kaur et al. [137] proposed a Multimodal Biometric System Using Speech and Signature Modalities.

\section{Structural aapproaches}

Structural approaches are divided into three categories string matching, structural description graph and tree matching. String matching approaches $[24,34,35,56,136,217]$ can be used for both verification and identification purposes. Structural description graph (SDG) approaches [54, 99, 101, 111] are used to verify the structural organization of a questioned signature. Tree matching approaches [176] involve building a tree of peaks and valleys of a signature. In this approach, two trees are built for the test signature and the reference signature, then the distance between them is computed in terms of minimum number of operations needed to transform one tree to the other. It was found that no technique was globally superior to the other two although regional correlation was often better and much faster[80]. Anil K. 
Jain et al. [24] proposed a DSV system using string matching. Two types of local features are extracted from the $x$ and $y$ coordinates: spatial and temporal. Local features of each signature are represented as a string at each sampling position. A customized DTW that includes a penalty for differences in the number of strokes is used to find dissimilarity values. Gupta and Joyce [35] described a technique which uses $x$ and y profile extrema (peaks or valleys) values to capture a signature's shape. $\mathrm{X}, \mathrm{Y}$, speed and acceleration profiles are calculated at each sampling point, then these profiles are divided into valleys and peaks. Each peak or valley in each profile is assigned a unique symbol. To compare two signature shapes, the string representations of the extrema for each are found and the two strings are then compared and distance between them is calculated. Texas Instruments [34] adopted this method and implemented it on the TMS320 Digital Signal Processors, after adding neural clustering techniques to enhance the discriminating power and arrive at a very simple and low-cost solution that can be embedded in existing pen-based systems, such as handheld computers and transaction units. Gupta and Joyce [56] presented four experiments to evaluate the performance of the technique in [35] and a number of its variations. Felicia Soedjianto et al. [136] proposed a DSV system based on dominant points of the signature. These dominant points are converted into codes which is used to determine the stroke direction. The code extracted from the input signature is then matched with the stored template. The alternation of the stroke direction will be converted into a number which is compared to a threshold. Sascha Schimke et al. [217] presented a new approach for DSV system based on event-string modeling of features derived from pen-position and pressure signals. The Levenshtein Distance which is a distance measure well known from textual pattern recognition, is used for comparison of signatures. the classification is carried out by applying a nearest neighbor classifier. Dimauro et al. [54] proposed a DSV system that finds the fundamental components of the test signature and then checks if these components appear in the sequence derived from the reference signatures using a knowledge-base that consists of the Component Reference Table and the Structural Description Graph. Kaiyue Wang et al. [111] proposed an on-line signature verification approach based on graph representation and graph matching techniques. Kai Huang and Hong Yan [101, 114] used the dynamic warping of the sequences of basic handwriting strokes in the reference signatures to deduce the stability and style-variation characteristics of the signer. The technique models each signature by a weighted Structure Description Graph (SDG) of the handwriting components. SDG was first used by Dimauro et al. $[54,96]$ to model handwriting strokes separated by pen-lifts. The stable handwriting features are emphasized. The style variations allows following all permissible paths within the SDGs. Parizeau and Plamondon [176] compared between three different signal matching algorithms for online signature verification: regional correlation, DTW, and skeletal tree matching (STM).

\section{Statistical-Based Approaches}

Most of existing online signature verification approaches involves preprocessing stages such as re-sampling, normalization and alignment of signatures in order to obtain robust signature features of equal length. Unfortunately, this process may cause unique properties of the user signatures to be lost. In the other hand, statistical analysis-based DSV systems [23, 42, 50, 102, 133, 154] avoid preprocessing step and still be able to develop a robust mechanism for detecting forgeries in the claimed signature. Herbst and Liu [53] proposed two approaches for segmentation: the first uses pen-down and pen-up occurrences within the signature and the second uses equal time components of the signature. Before applying the correlation test, the algorithm rejects any signature that is larger or smaller in time than $20 \%$ of the reference signature. This resulted in almost $60 \%$ of forgeries being rejected. Once passing this initial test, the correlation test is applied after the segments are shifted if necessary by up to $20 \%$ of the reference signature's segment duration, extra pen lifts are eliminated and correlation results are weighted to penalize excessive shifting, and weighted by the reference signature length of the segment since larger segments are believed to be harder to forge. Martinez-Diaz et al. [23] proposed a score normalization approach for DSV using Universal Background Model Bayesian adaptation to generate the client model. This Universal Background Model (UBM) is trained once and can be then used for all the claiming users. The UBM is chosen to be The Gaussian Mixture Model (GMM) trained with feature vectors from a pool of Users. In the verification process, the user signature is compared to his claimed template and the resulting score is normalized by its similarity to the UBM. The similarity between signature samples is computed using statistical pattern classification techniques. Hastie and Kishon [42] proposed a statistical model for DSV that assumes that the test signature consists of a reference signature which is transformed from occasion to occasion. The model consists of a template signature for each individual, and several factors controlling variations in each rendition of this template. The estimated template contains the mean of a sample of signatures from an individual as well as the variation factors that are used to estimate several measures of variance. Winston Nelson et al. [50] discussed three methods for on-line signature verification that are based on statistical models of features that summarize different aspects of signature shape and the dynamics of signature production. Rather than using detailed comparisons of shape and speed patterns of a test signature with the model of the reference signatures, the authors developed a decision criteria based on statistical models for the set of global features that summarize various aspects of a signature's shape and dynamics. Jonas Richiardi et al. [133] proposed a middle way between static discriminative classifiers and dynamic generative models, by using a static generative model together with specific feature extraction steps. They used a specific topology of static Bayesian network to perform classification of multivariate time series. Results showed that when using appropriate features extracted from the time series, the framework of static Bayesian networks offers an elegant approach to classification of multivariate time series. Hairong Lv and Wenyuan Wang [224] proposed a DSV system based on Dynamic Bayesian Network. Loris Nanni et al $[8,10]$ proposed a feature based DSV system based on Parzen window classifier (PWC). It used global analysis of input signatures and one-class classifiers. They reported that the fusion between Principal component analysis description and PWC drastically outperforms the state-of-the-art works using only PWC. Alisher Kholmatov and Berrin Yanikoglu [102] proposed a DSV system based on the Bayes classifier on the original data, as well as a linear classifier used in conjunction with Principal Component Analysis (PCA). Shern Cheng Yau [154] presented a comparison between Artificial 
Neural Network (ANN)-based and statistical analysis classifier-based DSV system and reported that statistical analysis produced better recognition rates compared to the ANN.

\section{Support Vector Machine}

Support vector machines (SVM) have been widely used to solve classification and regression problems. The main advantages of SVM are having a convex objective function with efficient training algorithms and good generalization properties. SVM use kernel functions to measure the similarity of two data objects, in DSV case, time series. However, standard kernel functions such as Gaussian or polynomial kernels are not well suited for time-series processing[80]. Yenwei Lee [182] compared between several classifiers for DSV such as DTW, ANN, HMM and SVM. The best result yield $95.8 \%$ verification accuracy rate by SVM modeling with Gaussian Radial Basis Function Kernel method. Marianela Parodi and Juan Carlos $[3,16]$ compared two classifiers, namely, Support Vector Machines and Random Forests that are used in the verification experiments and reported that the results obtained with RF are better than the ones obtained with SVM. Some of the DSV techniques that used SVM are Hong-Wei Ji and Zhong-Hua Quan[160], Fauziyah S. et al. [169], AitorMendaza-Ormaza et al. [170], Vadim Mottl et al. [201], Alexander Tatarchuk et al. [202], Valentina Sulimova et al. [213], Eugen Lupu et al. [107] and Yenwei Lee [182]. Christian Gruber et al. [177, 178] proposed a DSV technique that integrates a longest common subsequences (LCSS) detection algorithm which measures the similarity of signature time series into a kernel function for support vector machines (SVM). Eugen LUPU et al. [107] proposed a DSV system based on the TESPAR coding method and SVM classifier. this approach applies TESPAR coding to generate from the timebased functions that describe the local properties of the signature some global features stored in fixed dimensions matrices. Biswajit Kar and Pranab Dutta [179] proposed a DSV system based on SVM. The feature selection is performed by MRMR (Minimum Redundancy Maximum Relevance) method. The Verification is performed using global features and scores from functional features. These scores are considered as additional features in a two-class classification problem solved with the ANN and SVM. Verification accuracy is enhanced by fusion of user specific global and functional features.

\section{Neural Networks}

In order to learn the signature verification system to distinguish between genuine signatures and forgeries, we must provide it with samples of the two types. Since it is hard to define a class of forgers, some authors prefer to use genuine signatures that have been deformed instead. F. LECLERC and R. PLAMONDON [11] presented a comparison between neural network-based DSV systems that appear from 1989 to 1993. Many authors used the parametric approach to incorporate the usage of neural networks for the verification of online signatures. Tarig Osman [97] proposed a neural network-based online signature verification system using vector autoregressive modeling and a velocity segmentation scheme. Artur Szklarzewski and Marcin Derlatka[120] proposed an On-line Signature verification System based on the single-output multilayer perceptron (MLP). Daniel Oldham [164] proposed a DSV system based on time-delay neural network. Wan-Suck Lee [166], proposed a DSV system based on a multilayer perceptron neural network with a single hidden layer; where the inputs to the neural network are second order AR coefficients. Lina Mi [186] proposed a DSV system based on a three-layer feed-forward neural network with a Gaussian ridge activation function of neurons in hidden layer and output layer of neuro-template. P. Gentric and J. Minot use the signals $x(t), y(t)$ and $p(t)$ (the coordinates and pressure obtained by a special sensor) and elastic matching combined with dynamic programming to define four measures: mean pressure distortion, written shape distortion, dynamic difference and the ratio of signature durations[11]. M. MAILAH et al. [13] employed two multilayer feed-forward (MLF) neural network to train and verify the data. Tham Keit et al. [145] used time series modeling with autoregressive (AR) technique to calculate the AR coefficients from all segments of the signature. These coefficients are then used to train a multi-layer perceptron (MLP) neural network using the back propagation algorithm. Milton and Fernando [148] proposed a DSV system based on ANN. Julio Martínez-Romo et al. [171, 172] presented an on-line handwritten signature verification based on optimal feature representation (OFR) and neural-network-driven fuzzy reasoning (NND-FR). Alan McCabe et al. [173] proposed a DSV system using neural networks. Several static and dynamic features are extracted and used to train the neural network. Several Network topologies are tested and their accuracy is compared. Adam Czajka and Andrzej Pacut [174] proposed a DSV system based on neural networks. first, cluster analysis is applied to segment the feature space into sub-regions of "similar" signatures to facilitate the classification and verification tasks. Then, a two-layer sigmoidal perceptron was used to approximate the classification function. The authors also applied an adaptive radial-basis network called the RCE network. finally, the two networks are compared. Experimental results showed that, the RCE network failed in verification tasks.

\section{Transform Domain Approaches}

Time domain graph shows how a signal changes over time, whereas a frequency-domain graph shows how much of the signal lies within each given frequency band over a range of frequencies. The background and advantages of transform domains are beyond the scope of this work. This section only concentrates on the techniques that used transform domain. Transform domain approaches include Fourier descriptors-based approaches[68, 108, 116, 123, 130, 139], Discrete Wavelet Transform approaches [74, 76, 109, 110, 117, 125, 127, 130, 160, 183, 184, 185], Zernike moments [57], Linear Prediction Coding [40, 41], Mellin transform [100], Discrete Cosine Transform [47, 88, 144], Gabor [91, 92], Autoregressive [97, 103, 153] and Linear Programming Descriptor [47]. Fourier descriptors are invariant with respect to translation, rotation and magnification of the trace. Impedovo et al. [108] used the one-dimensional spectral analysis to establish the variability of a writer's signature in the frequency domain. Mingfu Zou et al. [116] presented a novel DSV approach using local shape Analysis. First, the input signature is segmented into several segments using HMM, then each two adjacent segments are combined to form a long segment and finally, the spectral and tremor information of this segment are calculated using Fast Fourier Transform (FFT). The test signature is accepted or rejected based on the similarity between 
its spectral and its prototype. Giovanni Dimauro [68] proposed a DSV system based on Fourier analysis. Fourier descriptors are used to extract a set of discriminant features that can be used to describe the shape of the signature. The verification system depends on one-dimensional spectral analysis of the strokes that separate 'writing units'. Kholmatov and Yanikoglu [139] presented a preliminary individuality model for online signatures using the Fourier domain representation of the signature. The normalized Fourier coefficients are used as a global features describing the signature.

Wavelets are a powerful statistical tool which can be used for a wide range of applications such as Signal processing, Data compression, Smoothing and image de-noising, Fingerprint verification, DNA analysis, protein analysis, Bloodpressure, heart-rate and ECG analyses, Finance, Internet traffic description, Industrial supervision of gear-wheel, Speech recognition and Computer graphics. DWT can be also used authenticating online signatures. Some of the techniques that used DWT for online signature verification are in $[74,76,109,110,117,125,127,130,160,183,184,185]$. Nakanishi et al. $[109,183,185]$ presented a DSV system based on the DWT and the adaptive signal processing. The same author [74] extracted features of pen-position and pen-movement angle by the sub-band decomposition using the DWT. Low frequency sub-band signals are used as features for suppressing the intra-class variation in the pen-movement angle. The same authors [76] proposed reducing the computational complexity of the DWT domain on-line signature verification by utilizing the pen-movement vector as an input parameter. Sánchez-Diaz et al. [110] proposed a DSV system based on applying a modified DTW algorithm to the signature wavelet coefficients. DWT is used to analyze the temporal and spectral properties of non-stationary signals. The modification on DTW relies on using direct matching points (DMP) information to dynamically adapt the similarity measure. Chang et al. [117] proposed a DSV system based on wavelet transform of global feature. Firstly, signature data were preprocessed using normalizing, de-bouncing and Gaussian filtering. Then wavelet transform was used for feature extraction. Five functions were used to represent the signatures. Each function was compressed using the Duabechies-4 wavelet. Zhang and Wu [125] proposed a DSV system that uses wavelet transform to extract characteristic points of signatures and builds 5-dimension feature sequences, then matches these sequences of test signature and its corresponding templates and fuse five decisions by majority voting strategy to make final decision. Wang et al. [127] proposed a DSV system using wavelet packet, which performs better than classical wavelet transform in majority of cases. In the DWT, only the approximation signal at each level is decomposed, without further operations on the detail signals. In order to allow decompositions of detail signals at each level, the same process of decomposing approximation signals can be applied to them. The resulting decomposition tree will be a complete binary tree since at each node, the signal is decomposed. This type of wavelet decomposition is called wavelet packet (WP). Hong-Wei Ji and Zhong-Hua Quan [160] proposed a DSV system based on Wavelet transform and SVM.

Radhika et al. [57] proposed a DSV system based on Zernike moments. The Zernike moments are a set of complex polynomials that are orthogonal on the unit circle. Zernike moments are orthogonally stable, rotation invariance and provide image reconstruction. A lower order Zernike moments are used to analyze the shape of the acceleration plot. The low order moments represent the global shape of a pattern and the higher order represents the detail. High order Zernike moments are not used because they are more sensitive to noise and take significantly longer time to compute. The acceleration values of an online signature are represented by a weighted sum of Zernike polynomials. Quen-Zong Wu et al. $[40,41]$ proposed a DSV system for Chinese character signatures based on Linear Prediction Coding (LPC) cepstrum and neural networks. Asghar Fallah et al. [100] proposed a hybrid approach for DSV based on combining Mellin transform, Mel Frequency Cepstral Coefficient (MFCC) and neural network. S. Rashidi et al. [88] presented a DSV system based on a Discrete Cosine Transform (DCT), which is applied to 44 time-based signals. Joan Fàbregas and Marcos FaundezZanuy [144] presented a DSV system based on DCT feature extraction with discriminability feature selection. Loris Nanni and Alessandra Lumini [47] proposed a DSV system based on local information and on a one-class classifier, the Linear Programming Descriptor (LPD) classifier. Jonghyon Yi et al. [91] proposed a DSV system where the Local temporal shifts existing in signatures are estimated by the differences of the phase outputs of Gabor filter applied to signature signals. Kekre and Bharadi [92] proposed a feature vector based on Gabor filtering for the DSV. Tarig Osman [97], N. Mohankrishnan [103], Yi Gu [153] proposed a DSV system based on Autoregressive Model Representation. K. Barkoula et al. [119] proposed a DSV system based on Turning Angle Sequence (TAS) representation and longest common subsequence matching. Christian O'Reilly and Réjean Plamondon [113] proposed a DSV system in which signature representation is performed using Sigma-Lognormal modeling. Jianbin Zheng et al. [196] applied discrete Fréchet distance to on-line signature verification using only coordinate position features of extreme point.

\section{Intelligent Approaches}

A large number of features can be extracted from the signature but not all these features are used in the verification system because some of them may be unfavorable for verification of particular signatures. The most important issues in the signature verification are how to select a reduced set of features that enhance the discriminative power of a signer and how can a legitimate analysis be made. Genetic algorithm (GA) is used to provide an effective solution for the problem of feature selection. GA can determine the type and the number of features to constitute the feature set for the signer that will maximize a suitably chosen discriminating function. The intelligence in this verification model is made up of fuzzy logic and genetic algorithm. The advantages of Fuzzy logic is its ability to solve complex problems by determining outputs of a given set of inputs without using conventional mathematical models. XUHUA et al. [82] proposed a new method to select the partial curves and features of the curves of signatures for verification using GA. The features are determined by chromosomes and the crossover points are determined by the order number of strokes. W. Wijesoma [62, 89] proposed a DSV system that uses the Genetic Algorithm (GA) to determine the optimal personalized features for each subject and Fuzzy Logic to transform the heuristics conceived by a human verifier to rules that govern the behavior of the DSV system. The fuzzy system takes in the total degree of match (TDOM) of shape and the TDOM of dynamics as its inputs. The output linguistic variable of the fuzzy block is the Degree Of Authenticity (DOA) of signature which is compared to a 
threshold to accept or reject the signature. Di Lecce et al. [66] proposed a multi-expert system for DSV that combines three experts by majority voting. The system consists of three experts. the first expert uses shape-based features and performs signature verification by a wholistic analysis. The second and third experts use speed-based features and performs signature verification by a regional analysis. The decisions of the three experts are combined by majority voting: if at least two decisions are "genuine" the final response is "genuine"; if at least two decisions are "false" the final response is "false"; otherwise the final response is "rejected". Ji Moon et al. [70] proposed a Multi-Confidential Levels for DSV using Data Mining Techniques. Two distance measures are computed. The feature-based approach uses the Canberra Distance (CD) to calculate the similarity between the input and the reference signature. Function-based signature verification method uses DTW to determine the similarity between reference signatures and the input signature. Jianbin and Guangxi [75] proposed a DSV system based on Evolutionary Computation (EC). Evolutionary Computation is a subfield of artificial intelligence that involves a metaheuristic or stochastic based continuous and combinatorial optimization methods which are mostly applied for black box problems. A. A. Zaher [77] proposed a robust hybrid technique for dsv using intelligent encoding of spatio temporal data. Joanna Putz-Leszczynska and Andrzej Pacut [195] proposed a DSV system based on a Global Classifier That Uses Universal Forgery Features. The idea of universal forgery features, where a global classifier is used to classify a signature as a genuine one or, as a forgery, without the actual knowledge of the signature template and its owner. This classifier is trained once on a group of historical data.

\section{Protected Approaches}

In this approaches, a set of noninvertible transformations are applied to the biometric template which can be represented by a set of sequences, in order to generate multiple transformed versions of the template. Once these transformations are made, retrieving the original data from the transformed template is computationally as hard as random guessing. Some of the techniques that used protected approaches are in $[61,130,132,188,189,190,191,211]$. Loris Nanni et al. [65] proposed a template protection scheme employing the BioHashing and the BioConvolving approaches. Manuel R. Freire $[189,190]$ presented a biometric template protection approach for dynamic signature verification that uses auxiliary (helper) data that allows the matching with secure templates but do not provide information to a potential attacker. Kuan et al. [130] proposed a method for secure computation of biometric hash on dynamic signatures using BioPhasor mixing and $2^{\wedge} \mathrm{N}$ discretization. Emanuele Maiorana et al. $[61,132]$ proposed a protected DSV system that uses BioConvolving to guarantee security and renewability of biometric templates and HMM-based matching strategy. The security and privacy issues still represent the main problems for the deployment of biometric-based authentication systems.

\section{Semi-Online Approaches}

Instead of using tablets for capturing online signatures, there are several techniques that used hand gloves [48,64, 134 $219]$ to capture dynamics of the hand during signing, or camera to track the signature production $[115,118,152159,165$, $180,181,204]$. Nidal Kamel et al. [48, 134, 219] used data glove as an input device and the singular value decomposition (SVD) numerical tool for modeling and matching. Another novel vision-based techniques for personal identification that uses a camera for tracking the signature is proposed by Munich and Perona [115, 118, 152 159, 165], KIRAN GURRALA [204], T. Venkatesh et al. [95] and Daigo Muramatsu et al.[180, 181]. The system falls within the category of on-line systems since the visual tracker of handwriting captures the timing information in the generation of the signature. The drawback of the technique is that if signatures have extremely fast strokes, the system loses track of the pen tip, and if users were asked to sign more slowly, the consistency in the signing behavior will be lost.

\section{REFERENCES}

[1] H. Lei, V. Govindaraju, "A comparative study on the consistency of features in on-line signature verification", Pattern Recognition Letters, Vol. 26, PP. 2483-2489, 2005.

[2] M. Martinez-Diaz, J. Fiérrez-Aguilar, J. Galbally, J. Ortega-Garcia, "Towards mobile authentication using dynamic signature verification: Useful features and performance evaluation", ICPR, 2008.

[3] M. Parodi and J. C. Gomez, "Online Signature Verification Based on Legendre Series Representation. Consistency Analysis of Different Feature Combinations", CIARP 2012, LNCS 7441, PP. 715-723, 2012.

[4] J. Richiardi, H. Ketabdar, A. Drygajlo, "Local and global feature selection for on-line signature verification", In Proc. IAPR 8th International Conference on Document Analysis and Recognition (ICDAR), 2005.

[5] J. J. Igarza, L. Gómez, I. Hernáez and I. Goirizelaia, "Searching for an Optimal Reference System for On-Line Signature Verification Based on (x, y) Alignment", ICBA 2004, LNCS 3072, PP. 519-525, 2004.

[6] A. A. Kholmatov, "Biometric identity verification using on-line and off-line signature verification," Master's thesis, Sabanci University, Istanbul, Turkey, 2003.

[7] M. Tanaka, Y. Ishino, H. Shimada, T. Inoue and A. Bargiela, "determination of decision boundaries for online signature verification", KES2003, LNAI 2773, PP. 401-407, 2003.

[8] L. Nanni, "Experimental comparison of one-class classifiers for online signature verification", Neurocomputing Vol. 69, PP. 869$873,2006$.

[9] C.E. Pippin, "Dynamic signature verification using local and global features", Technical report, Georgia Inst. Inform. Technol., Atlanta, GA, 2004.

[10] L. Nanni and A. Lumini, "ensemble of parzen window classifiers for on-line signature verification", Neurocomputing 68, PP. 217224, 2005. 
[11] F. Leclerc and R. Plamondon, "Automatic signature verification: the state of the art-1989-1993", Intl. Journal of Pattern Recognition and Artificial Intelligence, Vol. 8, No. 3, PP. 643-660, 1994.

[12] L. L. Lee, T. Berger, and E. Aviczer, "Reliable on-line human signature verification systems", IEEE Trans. Pattern Anal. Mach. Intell. (T-PAMI), Vol. 18, No. 6, PP. 643-647, Jun. 1996.

[13] M. Mailah and L. Boon Han, "Biometric signature verification using pen position, time, velocity and pressure parameters", Jurnal Teknologi A (48A), PP. 35-54, 2008.

[14] D. Sakamoto, H. Morita, T. Ohishi, Y. Komiya, and T. Matsumoto, "On-line signature verification algorithm incorporating pen position, pen pressure and pen inclination trajectories", Proc. ICASSP, PP. 993-996, 2001.

[15] M. Faundez-Zanuy, "On-line signature recognition based on VQ-DTW", Pattern Recognition, Vol. 40, No. 3, PP.981-992, 2007.

[16] M. Parodi, J. Gómez and M. Liwicki, "Online Signature Verification Based on Legendre Series Representation. Robustness Assessment of Different Feature Combinations", ICFHR, 2012.

[17] H. Feng and C. C. Wah, "Online signature verification using a new extreme points warping technique", Pattern Recognit. Lett., Vol. 24, No. 16, PP. 2943-2951, Dec. 2003.

[18] A. Kholmatov and B. Yanikoglu, "Identity authentication using improved online signature verification method", Pattern Recognit. Lett., Vol. 26, PP. 2400-2408, Nov. 2005.

[19] J. Fierrez-Aguilar, J. Ortega-Garcia, D. Ramos, and J. G.-Rodriguez, "HMM-based on-line signature verification: Feature extraction and signature modelling", Pattern Recognit. Lett.,Vol. 28, No. 16, PP. 2325-2334, Dec. 2007.

[20] A. McCabe, "Hidden Markov Modelling with Simple Directional Features for Effective and Efficient Handwriting Verification", 6th Pacific Rim International Conference on Artificial Intelligence, Melbourne, Australia, 2000.

[21] S. Garcia-Salicetti, N. Houmani and B. Dorizzi, "Novel Criterion for Writer Enrolment Based on a Time-Normalized Signature Sample Entropy Measure", EURASIP Journal on Advances in Signal Processing, 2009.

[22] J. F-Aguilar, L. Nanni, J. L-Penalba, J. O-Garcia, and D. Maltoni, "An on-line signature verification system based on fusion of local and global information", LNCS 3546, (AVBPA), New York, PP. 523-532., Jul. 2005.

[23] M. Martinez-Diaz, J. Fierrez and J. O-Garcia, "Universal Background Models for Dynamic Signature Verification", In proceedings IEEE Conference on Biometrics: Theory, Applications and Systems, BTAS, PP. 1-6, 2007.

[24] A. K. Jain, F. D. Griess, S. Connell, "On-line signature verification", Patt. Recogn., Vol. 35, PP. 2963-2972, 2002.

[25] V. S. Nalwa, "Automatic on-line signature verification", Proc. IEEE, Vol. 85, No. 2, PP. 215-239, Feb. 1997.

[26] G. K. Gupta, "The State of the Art in On-line Handwritten Signature Verification", Monash Univ., Australia, 2006.

[27] J. Richiardi and A. Drygajlo, “Gaussian Mixture Models for Online Signature Verification”, Speech Processing Group Signal Processing Institute Swiss Federal Institute of Technology, WBMA'03, California, PP.771-779, 2003.

[28] H. D. Chang, J. F. Wang, and H. M. Suen, "Dynamic handwritten Chinese signature verification", in Proc. 2nd Int. Conf. Doc. Anal. Recognit. (ICDAR-2), Tsukuba, Japan, Oct. PP. 258-261, 1993.

[29] J. Fierrez-Aguilar, J. Ortega-Garcia, and J. Gonzalez-Rodriguez, "Target Dependent Score Normalization Techniques and Their Application to Signature Verification", ICBA 2004, LNCS 3072, PP. 498-504, 2004.

[30] V. Ly, S. Salicetti and B. Dorizzi, "On Using the Viterbi Path Along With HMM Likelihood Information for Online Signature Verification", IEEE Transact. on Systems, Man, and Cybernetics, Vol. 37, No. 5, PP.1237-1247, 2007

[31] A. G. Reza, H. Lim and M. J. Alam, "An Efficient Online Signature Verification Scheme Using Dynamic Programming of String Matching", ICHIT 2011, LNCS 6935, PP. 590-597, 2011.

[32] E.-Mari. Nel, "estimating the pen trajectories of static handwritten scripts using hidden markov models", PHD thesis, University of Stellenbosch, Dec. 2005

[33] S. H. Kim et al., "applying personalized weights to a feature set for on-line signature verification" in proceedings of the Intl. Conference on Document Analysis and Recognition, Vol. 2, PP. 882-885, 1995.

[34] H. Dullink, B. Daalen, J. Nijhuis, L. Spaanenburg, H. Zuidhof, "Implementing a DSP Kernel for Online Dynamic Handwritten Signature Verification using the TMS320 DSP Family", EFRIE, France SPRA304, December 1995.

[35] G. K. Gupta and R. C. Joyce, "A Study of Shape in Dynamic Handwritten Signature Verification", Technical Report 97/04, Computer Science Department, James Cook University of North Queensland, 1997.

[36] J. Csirik, Z. Gingl and E. Griechisch, "The effect of training data selection and sampling time intervals on signature verification", Proceedings of the 1st International Workshop on (AFHA), 2011.

[37] L. YANG, B. K. WIDJAJA and R. PRASAD, "Application of hidden markov models for signature verification", Pattern Recognition, Vol. 28, No. 2, PP. 161-170, 1995.

[38] J. G. A. Dolfing, E. H. L. Aarts, and J. J. G. M. Van Oosterhout, "On-line verification signature with hidden Markov models," in Proc. 14th Int. Conf. Pattern Recog. (ICPR-14),Brisbane, Australia, PP. 1309-1312, Aug. 1998.

[39] G. Rigoll and A. Kosmala, "A systematic comparison between on-line and offline methods for signature verification with hidden Markov models", in Proc. 14th Int. Conf. Pattern Recog. (ICPR-14), Vol. 2, PP. 1755-1757, 1998.

[40] Q. Zong Wu, I. Chang Jou, and S. Yin Lee, "On-Line Signature Verification Using LPC Cepstrum and Neural Networks", IEEE TRANSACT. ON SYSTEMS, MAN, AND CYBERNETICS, VOL. 27, NO. 1, PP. 148-153, 1997. 
[41] Q. ZONG WU, S. YIN LEE and I. CHANG JOU, "on-line signature verification based on logarithmic spectrum", Pattern Recognition, Vol. 31, No. 12, PP. 1865-1871, 1998.

[42] T. Hastie and E. Kishon, "A model for signature verification", Murray Hill, Monash University, Australia, 1992.

[43] J. Ortega-Garcia, J. Fierrez-Aguilar, J. Martin-Rello, and J. Gonzalez-Rodriguez, "Complete signal modeling and score normalization for function-based dynamic signature verification", LNCS 2688, PP. 658-667, 2003.

[44] T. Qu, A. El Saddik and A. Adler, "a stroke based algorithm for dynamic signature verification", CCECE 2004 - CCGEI 2004, Niagara Falls, May 2004.

[45] Z. Hua Quan and H. wei Ji, "Aligning and Segmenting Signatures at Their Crucial Points Through DTW", ICIC 2005, Part I, LNCS 3644, PP. 49-58, 2005.

[46] D. Muramatsu, M. Kondo, M. Sasaki, S. Tachibana, and T. Matsumoto, "A Markov chain Monte Carlo algorithm for Bayesian dynamic signature verification," IEEE Trans. Inf. Forensics Secur.,Vol. 1, No. 1, PP. 22-34, Mar. 2006.

[47] L. Nanni and A. Lumini, "a novel local on-line signature verification system", Patt. Recog. Lett. 29, PP.559-568, 2008.

[48] N. S. Kamel, S. Sayeed and G. A. Ellis, "Glove-Based Approach to Online Signature Verification", IEEE Transactions On Pattern Analysis And Machine Intelligence, VOL. 30, NO. 6, PP. 1109-1113, JUNE 2008.

[49] H. Ketabdar, J. Richiardi, and A. Drygajba, "Global feature selection for on-line signature verification," in Proc. Conf. Int. Graphonomics Soc. (IGS 2005),Salerno, Italy, Jun. 26-29, PP. 59-63, 2005.

[50] W. Nelson, W. Turin, and T. Hastie, "Statistical methods for on-line signature verification," Int. J. Pattern Recognit. Artif. Intell. (IJPRAI), Vol. 8, No. 3, PP. 749-770, 1994.

[51] B. Yanikoglu and A. Kholmatov, "An improved decision criterion for genuine/forgery classification in on-line signature verification", Presented at ICANN/ICONIP Workshop on Biometrics", June 2003.

[52] G. K. Gupta and R. C. Joyce, "A study of some pen motion features in dynamic handwritten signature verification", Department of Computer Science, James Cook University, Townsville, Australia, 1997.

[53] N. Herbst and C. Liu, "Automatic signature verification based on accelerometry", IBM Vol. 21, PP. 245-253, 1977.

[54] G. Dimauro, S. Impedovo and G. Pirlo, "Component-oriented algorithms for signature verification", Int. J. Pattern Recog. Artif. Intell. Vol.8, No. 3 PP. 771-794, 1994.

[55] R. S. Kashi, J. Hu, W. L. Nelson and W.Turin, "On-line handwritten signature verification using Hidden Markov Model features", Proc. of the 4th Intl. Conf. on Document Analysis and Recognition, Vol. 1, PP. 253-257, 1997.

[56] G.K. Gupta and R.C. Joyce, "Using position extrema points to capture shape in on-line handwritten signature verification", Pattern Recognition, Vol. 40, PP. 2811-2817, 2007.

[57] K. R. Radhika, M. K. Venkatesha, G. N. Sekhar, "An approach for on-line signature authentication using Zernike moments", Pattern Recognition Letters, Vol. 32, No. 5, PP. 749-760, 2011.

[58] N. Houmani, S. Garcia-Salicetti, B. Dorizzi and M. El-Yacoubi, "On-line Signature Verification on a Mobile Platform", MOBICASE 2010, LNICST 76, PP. 396-400, 2012.

[59] J. F-Aguilar, S. Krawczyk, J. O-Garcia and A. K. Jain, "Fusion of local and regional approaches for on-line signature verification", Proc. IWBRS, PP. 188-196, Beijing, China, October 2005.

[60] M. Kondo, D. Muramatsu, M. Sasaki, T. Matsumoto, "A bayesian MCMC On-line Signature Verification", LNCS, PP. 540-548, 2003.

[61] E. Maiorana, P. Campisi, J. Fierrez, J. Garcia, and A. Neri,"cancelable templates for sequence-based biometrics with application to on-line signature recognition", IEEE transactions on systems, man, and cybernetics, VOL.40, NO.3, PP.525-538, 2010.

[62] W. Sardha Wijesoma, M. Mingming and K.W. Yue, "On-Line Signature Verification Using a Computational Intelligence Approach", Fuzzy Days, LNCS 2206, PP. 699-711, 2001.

[63] A. Humm, J. Hennebert, and R. Ingold, "gaussian mixture models for chasm signature verification", MLMI, LNCS 4299, 2006.

[64] A. Samraj, S. Sayeed, L. C. Kiong, N. E. Mastorokis, "eliminating forgers based on intra trial variability in online signature verification using handglove and photometric signals", Journal of Information Security, PP. 23-28, 2010.

[65] L. Nanni, E. Maiorana, A. Lumini and P. Campisi, "Combining local, regional and global matchers for a template protected on-line signature verification system", Expert Systems with Applications Vol.37, PP. 3676-3684, 2010.

[66] V. Di Lecce, G. Dimauro, A. Guerriero, S. Impedovo, G. Pirlo, and A. Salzo, "A Multi-expert System for Dynamic Signature Verification", MCS, LNCS 1857, PP. 320-329, 2000.

[67] G. Dimauro, S. Impedovo, R. Modugno, G. Pirlo, and L. Sarcinella, "analysis of stability in hand-written dynamic signatures", Proc. IWFHR-8,Niagara-on-the-Lake, ON, Canada, PP. 259-263, Aug. 2002.

[68] G. Dimauro, "Fourier Transform in Numeral Recognition and Signature Verification", Pattern Recognition, Machine Intelligence and Biometrics Book Part IV, PP. 823-857, 2011.

[69] J. Shin and T. Takanashi, "On-line Signature Verification based on Pen Inclination and Pressure Information", International Journal of Electrical and Computer Engineering (IJECE), Vol.2, No.4, PP. 441-446, August 2012.

[70] J. H. Moon, S.-Gyu Lee, S. Y. Cho and Y.-Sung Kim, "A hybrid online signature verification system supporting multi-confidential levels defined by data mining techniques", IJISTA, 9(3/4), PP.262-273, 2010. 
[71] G. Congedo, G. Dimauro, A. M. Forte, S. Impedovo, and G. Pirlo, "Selecting reference signatures for on-line signature verification", ICIAP-8, LNCS, Vol. 974, Berlin, Germany: Springer-Verlag, PP. 521-526, Sep. 1995.

[72] S. Krawczyk and A.K. Jain, "Securing Electronic Medical Records Using Biometric Authentication", AVBPA, PP.1110-1119, 2005.

[73] D. Muramatsu and T. Matsumoto, "An HMM On-line Signature Verifier Incorporating Signature Trajectories", ICDAR, 2003.

[74] I. Nakanishi, H. Sakamoto, N. Nishiguchi, Y. Itoh, and Y. Fukui, "Multi-Matcher On-Line Signature Verification System in DWT Domain", presented at IEICE Transactions, PP.178-185, 2006.

[75] Z. Jianbin and Z. Guangxi, "A New Algorithm for On-Line Handwriting Signature Verification Based on Evolutionary Computation", WUJNS, Vol. 11, No. 3, PP. 596-600, 2006.

[76] I. Nakanishi, H. Sakamoto, N. Nishiguchi, Y. Itoh, and Y. Fukui, "DWT Domain On-Line Signature Verification Using the PenMovement Vector", presented at IEICE Transactions, PP.1129-1131, 2006.

[77] A. A. Zaher and A. Abu-Rezq, "a robust hybrid technique for signature verification using intelligent encoding of spatio temporal data", ICIC Vol. 7, No. 4, PP. 1789-1818, April 2011.

[78] W. Chang and J. Shin, "DPW Approach for Random Forgery Problem in Online Handwritten Signature Verification", in Proc. NCM (1), PP. 347-352, 2008.

[79] Tong Qu, "Dynamic Signature Verification System Design Using Stroke Based Feature Extraction Algorithm", Master thesis, Ottawa, Ontario, Canada 2004.

[80] D. Impedovo and G. Pirlo, "automatic signature verification: the state of the art", IEEE TRANSACTIONS ON SYSTEMS, MAN, AND CYBERNETICS, VOL. 38, NO. 5, PP. 609-635, SEPTEMBER 2008.

[81] J. Zhang and S. KAMATA, "Online Signature Verification Using Segment-to-Segment Matching", In: Int. Conf. on Frontiers in Handwriting Recognition ICFHR, 2008.

[82] Y. Xuhua, T. Furuhashi, K. Obata, and Y. Uchikawa, "Selection of features for signature verification using the genetic algorithm", Comput. Ind. Eng., Vol. 30, No. 4, PP. 1037-1045, 1996.

[83] W. Chang and J. Shin, "Dynamic Position Warping for On-line 2D Shape," 13th Conference of the International Graphonomics Society, Melbourne, Australia, PP.36-39, Nov., 2007.

[84] H. Bunke, J. Csirik, Z. Gingl and E. Griechisch, "Online Signature Verification Method Based on the Acceleration Signals of Handwriting Samples", CIARP 2011, LNCS 7042, PP. 499-506, 2011

[85] J. Lee, H.-S. Yoon, J. Soh, B. T. Chun, and Y. K. Chung, "Using geometric extrema for segment-to-segment characteristics comparison in on-line signature verification," Pattern Recognit., Vol. 37, No. 1, PP. 93-103, 2004.

[86] V. Di Lecce, G. Dimauro, A. Guerriero, S. Impedovo, G. Pirlo, A. Salzo and L. Sarcinella, "selection of reference signatures for automatic signature verification", ICDAR-5, Bangalore, India, PP. 597-600, 1999.

[87] R. Kashi, J. Hu, W. Nelson, W. Turin, "A Hidden Markov Model approach to online handwritten signature verification", IJDAR, 1998.

[88] S. Rashidi, A. Fallah and F. Towhidkhah, "Feature extraction based DCT on dynamic signature verification", scientia iranica, transactions computer science \& engineering and electrical engineering, 2012.

[89] W. S. Wijesoma, K.W. Yue, K.L. Chien and T.K. Chow, "Online Handwritten Signature Verification for Electronic Commerce over the Internet", WI 2001, LNAl 2198, PP. 227-236, 2001.

[90] M. M. Shafiei and H. R. Rabiee, "A new on-line signature verification algorithm using variable length segmentation and hidden Markov models", ICDAR-7, IEEE Comput. Soc.,Edinburgh, U.K., Vol. 1, PP. 443-446, Aug. 2003.

[91] J. Yi, C. Lee, and J. Kim, "Online signature verification using temporal shift estimated by the phase of Gabor filter", IEEE Trans. Signal Process., Vol. 53, No. 2, PP. 776-783, Feb. 2005.

[92] H. B. Kekre and V. A. Bharadi, "gabor filter based feature vector for dynamic signature recognition", international journal of computer applications (0975 - 8887) Vol. 2, No.3, May 2010.

[93] Z. Wu, P. Fang and F. Shen, "Online Signature Verification Based on Global Feature of Writing Forces", ICB 2006, LNCS 3832 , PP. 516-522, 2005.

[94] M. C. Fai rhust AND S. Ng, "Management of access through biometric control A case study based on automatic signature verification", UAIS, PP. 31-39, 2001.

[95] T. Venkatesh, S. Balaji and A. S. Chakravarthy, "security evaluation of online signature verification system using webcams", international journal of computer applications, Vol. 41, No. 15, March 2012.

[96] G. Dimauro, S. Impedovo, and G. Pirlo, "a stroke-oriented approach to signature verification", in from pixels to features iii-frontiers in handwriting recognition, PP. 371-384, 1992.

[97] O. T. Abd-Elgadir-Mohammed, "a neural-network-based online signature verification system using vector autoregressive modeling and a novel velocity segmentation scheme", PHD thesis, Univ. of Detroit Mercy, 2009.

[98] G. Dimauro, S. Impedovo, G. Pirlo, "A Signature Verification System Based on a Dynamical Segmentation Technique", Proceedings of IWFHR III, Buffalo, New York, USA, PP. 262-271, 1993.

[99] L. Bovino, S. Impedovo, G. Pirlo, and L. Sarcinella, "multi-expert verification of hand-written signatures", ICDAR-7, IEEE Comput. Soc.,Edinburgh, U.K., PP. 932-936, Aug. 2003.

[100] A. Fallah, M. Jamaati and A. Soleamani, "a new online signature verification system based on combining mellin transform, mfcc 
and neural network", Digital Signal Processing, Vol. 21, No. 2, PP. 404-416, March 2011.

[101] K. Huang and H. Yan, "Stability and style-variation modeling for on-line signature verification", Pattern Recognit., Vol. 36, No. 10, PP. 2253-2270, Oct. 2003.

[102] A. Kholmatov, B. Yanikoglu, "Biometric Authentication Using Online Signatures", ISCIS, PP. 373-380, 2004.

[103] N. Mohankrishnan, M. J. Paulik and M. Khalil, "on-line signature verification using a nonstationary autoregressive model representation", ISCAS, PP. 2303-2306, 1993.

[104] J. W. Kim, "various implementations of advanced dynamic signature verification system", Advances in Computer Science, Eng. \& Appl., AISC 166, PP. 203-210, 2012.

[105] J.J. Igarza, I. Goirizelaia, K. Espinosa, I. Hernáez, R. Méndez, and J. Sánchez, "online handwritten signature verification using hidden markov models", in Proc. CIARP, PP.391-399, 2003.

[106] R. Martens and L. Claesen, "Incorporating local consistency information into the online signature verification process", presented at IJDAR, PP.110-115, 1998

[107] E. LUPU, S. EMERICH and F. Beaufort, "online signature recognition using a global features fusion approach", ACTA Technica Napocensis Electronics and Telecommunications, Vol. 50, No. 3, PP. 13-20, 2009.

[108] S. Impedovo, M. Castellano, G. Pirlo and A. Mingolla ,"a spectral analysis-based signature verification system", Lecture Notes in Computer Science : Recent Issues in Pattern Analysis and Recognition, PP. 316-323, 1989.

[109] I. Nakanishi, N. Nishiguchi, Y. Itoh, and Y. Fukui, "On-Line Signature Verification Based on Discrete Wavelet Domain Adaptive Signal Processing", in Proc. ICBA, PP.584-591, 2004.

[110] J.C. Sánchez-Diaz, J.M. Ramírez-Cortés, R.A. Enriquez-Caldera and P. Gómez-Gil, "on-line signature verification based on modified dynamic time warping and wavelet sub-band coding", in Proc. MCPR, PP. 210-219, 2010.

[111] K. Wang, Y. Wang and Z. Zhang, "on-line signature verification using graph representation", sixth international conference on image and graphics, PP. 943-948, 2011.

[112] M. H. Mohammadi and K. Faez, "matching between important points using dynamic time warping for online signature verification", Cyber Journals: Multidisciplinary Journals in Science and Technology, JBIO, 2012.

[113] C. O'Reilly and R. Plamondon, "Development of a Sigma-Lognormal Representation for On-Line Signatures", Pattern Recognition, Vol. 42, PP. 3324-3337, 2009.

[114] K. Huang and H. Yan, "On-line signature verification based on stroke matching", DICTA2002: Digital Image Computing Techniques and Applications, Melbourne, Australia, Jan. 2002.

[115] M.E. Munich and P.Perona, "camera-based id verification by signature tracking", proc. ECCV, PP.782-796, 1998.

[116] M. Zou, J. Tong, C. Liu, and Z. Lou, "On-line signature verification using local shape analysis," in Proc. 7th Int. Conf. Doc. Anal. Recognit.(ICDAR-7), Vol. 1, Edinburgh, U.K., PP. 314-318, Aug. 2003.

[117] H. Chang, D. Dai, P. Wang, Y. Xu, F. Si and S. Huang, "Online Signature Verification Using Wavelet Transform of Feature Function", JOICS 9:11, PP. 3135-3142, 2012.

[118] M.E. Munich and P. Perona, "continuous dynamic time warping for translation-invariant curve alignment with applications to signature verification", Proc. ICCV, PP.108-115, 1999.

[119] K. Barkoula, G. Economou and S. Fotopoulos, "online signature verification based on signatures turning angle representation using longest common subsequence matching",IJDAR, Springer-Verlag Berlin Heidelberg, 2012.

[120] A. SZKLARZEWSKI and M. DERLATKA, "on-line signature biometric system with employment of single-output multilayer perceptron", Biocybernetics and Biomedical Engineering, Vol. 26, No. 4, PP. 91-102, 2006.

[121] M. Wirotius, J. Ramel, and N. Vincent, "improving dtw for online handwritten signature verification", ICIAR, PP.786-793, 2004.

[122] J. Putz-Leszczynska, M. Kudelski, "Hidden Signature for DTW Signature Verification in Authorizing Payment Transactions", Journal of Telecommunication and Information Technology 4, PP. 59-67, 2010.

[123] B. Yanikoglu and A. Kholmatov, "ONLINE SIGNATURE VERIFICATION USING FOURIER DESCRIPTORS", EURASIP Journal on Advances in Signal Processing, 2009.

[124] D. U. Cho, Y. L. Bae and I.S. Ko, "The Modified DTW Method for On-Line Automatic Signature Verification", NPC 2005, LNCS 3779, PP. 335-342, 2005

[125] L. Zhang and Z.Wu, "On-Line Signature Verification Based on Wavelet Transform to Extract Characteristic Points", ICIC, LNCIS 345, PP. 1002-1007, 2006.

[126] M. Bashir, J. Kempf, G. Schickhuber, G. Scharfenberg, "Online Person Authentication Using Dynamic Signature on a Novel Tactile and Pressure Sensitive Pad" in November 24-26, Telfor Belgrade, Serbia, 2009.

[127] K. Wang, Y. Wang, and Z. Zhang, "On-line signature verification using wavelet packet", Proc. IJCB, PP. 1-6, 2011.

[128] D. Lin and Y. Liao, "on-line handwritten signature verification using hidden semi-markov model", $\mathrm{HCl}$ (22), PP.584-589, 2011.

[129] P. Fang, Z. Wu, F. Shen, Y. Ge and B. Fang, "Improved DTW Algorithm for Online Signature Verification Based on Writing Forces", ICIC 2005, Part I, LNCS 3644, PP. 631-640, 2005.

[130] Y.W. Kuan, A.J. Teoh, and D.L. Ngo, "secure hashing of dynamic hand signatures using wavelet-fourier compression with 
biophasor mixing and 2n discretization", presented at EURASIP J. Adv. Sig. Proc., 2007.

[131] Colin Sindle, "Handwritten Signature Verification Using Hidden Markov Models", Master thesis, Nov. 2003.

[132] E. Maiorana, M. Martinez-Diaz, P. Campisi , J. O-Garcia, A. Neri, "template protection for hmm-based on-line signature authentication", IEEE Conference on Computer Vision and Pattern Recognition, June 2008.

[133] J. Richiardi, K. Kryszczuk, and A. Drygajlo, "static models of derivative-coordinates phase spaces for multivariate time series classification: an application to signature verification", Proc. ICB, PP.1200-1208, 2009.

[134] S. Sayeed, N. S. Kamel and R. Besar, "a novel approach to dynamic signature verification using sensor-based data glove", American Journal of Applied Sciences 6 (2), PP. 233-240, 2009.

[135] Z.-Hua Quan and K.-Hong Liu, "online signature verification based on the hybrid HMM/ANN Model", IJCSNS International Journal of Computer Science and Network Security, VOL.7, No.3, March 2007.

[136] F. Soedjianto, L. D. Kristianto and R. Adipranata, "signature recognition with dominant point method", Petra Christian University Surabaya, ICSIIT, 2007.

[137] M. Kaur, A. Girdhar and M. Kaur, "multimodal biometric system using speech and signature modalities", International Journal of Computer Applications (0975 - 8887), Vol. 5, No.12, August 2010.

[138] M. Martinez-Diaz, J. Fierrez and J. O-Garcia, "incorporating signature verification on handheld devices with user-dependent Hidden Markov Models", Proc. ICFHR, Montreal, Canada, August 2008.

[139] A. Kholmatov and B. Yanikoglu, "an individuality model for online signatures using global fourier descriptors", Biometric Technology for Human Identification V, Proc. of SPIE Vol. 6944, 694407, 2008.

[140] R. Martens and L. Claesen, "On-Line Signature Verification by Dynamic Time-Warping", Proc. 13th Int'I Conf. Pattern Recognition, PP. 38-42, 1996.

[141] R. Plamondon and G. Lorette, "Automatic signature verification and writer identification-the state of the art", Pattern Recognition, Vol. 1, No. 2, PP. 107-131, 1989.

[142] M.I. Khalil, M. Moustafa and H.M. Abbas, "Enhanced DTW based on-line signature verification", Proc. ICIP, PP. 2713-2716, 2009.

[143] M. Wirotius, J. Ramel and N. Vincent, "Selection of points for on-line signature comparison", Proc. IWFHR, PP.503-508, 2004.

[144] J. Fàbregas and M. Faundez-Zanuy, "On-line signature verification system with failure to enrol management", Pattern Recognition Vol. 42, PP. 2117-2126, 2009.

[145] T.H. Keit, R. Palaniappan, P. Raveendran and F. Takeda, "signature verification system using pen pressure for internet and ecommerce application", ISSRE, Hong Kong, Nov. 2001.

[146] B. Li, K. Wang, D. Zhang, "On-Line Signature Verification Based on PCA (Principal Component Analysis) and MCA (Minor Component Analysis)", ICBA, PP.540-546, 2004.

[147] B. Li, D. Zhang and K. Wang, "Improved Critical Point Correspondence for On-line Signature Verification", International Journal of Information Technology, Vol.12, No.7, 2006.

[148] M.R. Heinen and F.S. Osório, "handwritten signature authentication using artificial neural networks", in Proc. IJCNN, PP.50125019, 2006.

[149] V. Ly, S. Garcia-Salicetti, and B. Dorizzi, "fusion of HMM's likelihood and viterbi path for on-line signature verification", in Proc. ECCV Workshop BioAW, PP.318-331, 2004.

[150] A. Humm, J. Hennebert, and R. Ingold, "Combined Handwriting and Speech Modalities for User Authentication", presented at IEEE Transactions on Systems, Man, and Cybernetics, Part A, Vol. 39, No. 1, PP.25-35, Jan. 2009.

[151] A. Humm, J. Hennebert, and R. Ingold, "Spoken signature for user authentication", presented at J. Electronic Imaging, 2008.

[152] M.E. Munich and P. Perona, "Visual Identification by Signature Tracking", presented at IEEE Trans. Pattern Anal. Mach. Intell., PP.200-217, 2003.

[153] Y. Gu, "APPROACHING REAL TIME DYNAMIC SIGNATURE VERIFICATION FROM A SYSTEMS AND CONTROL PERSPECTIVE", Master thesis, Univ. of the Witwatersrand, Johannesburg, Sep. 2003.

[154] S. Cheng Yau, "Validation of Dynamic Signature for Identity Verification", PHD thesis, Telecommunication Engineering, RMIT University, Melbourne, Australia, Aug. 2008.

[155] D. Muramatsu and T. Matsumoto, "an HMM on-line signature verification algorithm", AVBPA, PP.233-241, 2003.

[156] J. MONTALVÃO, N. HOUMANI and B. DORIZZI, "comparing GMM and Parzen in automatic signature recognition a step backward or forward", Anais, ISSN, PP. 4463-4468,2010

[157] A. Humm, J. Hennebert and R. Ingold, "Hidden Markov Models for spoken signature verification", In Proc. of 1st IEEE Intl. Conf. on Biometrics: Theory, Applications, and Systems, BTAS, PP. 1-6, 2007.

[158] Y. Qiao and X. Wang and C. Xu "learning Mahalanobis distance for DTW based online signature verification", Proceeding of the IEEE International Conference on Information and Automation Shenzhen, PP. 333-338, 2011.

[159] M.E. Munich and P. Perona, "Visual Signature Verification Using Affine Arc-Length", CVPR, PP.2180-2186, 1999.

[160] H. Ji and Z. Quan, "Signature Verification Using Wavelet Transform and Support Vector Machine", ICIC (1), PP.671-678, 2005. 
[161] G. Taherzadeh, R. Karimi, A. Ghobadi, P. Vahdani Amoli and S. Mirjalili, "Categorizing Global and local features of On-line signature verification using DTW and Fuzzy logic", Multimedia University, Selangor, Malaysia.

[162] G. K. Gupta, R. C. Joyce, "A STUDY OF SOME GLOBAL FEATURES IN ONLINE HANDWRITTEN SIGNATURE VERIFICATION", Faculty of information technology, Monash University, 2006.

[163] H. Lei, S. Palla, and V. Govindaraju, "ER: an intuitive similarity measure for on-line signature verification", in Proc. IWFHR, PP.191-195, 2004.

[164] D. R. Oldham, "Biometric identification for dynamic signature verification using time delay neural networks", PHD thesis, Department of electrical engineering and computer science, Case Western Reserve University, Jan. 2001

[165] M. E. Munich and P. Perona, "Visual-based ID verification by signature tracking", Proc. of the 2nd International Conference on Audio- and Video-based person authentication (AVBPA99), Washington, March 1999.

[166] W.-Suck Lee, "A neural network-based on-line signature verification system", PHD thesis, University of Detroit Mercy, Oct. 1998.

[167] G. Congedo, G. Dimauro, S. Impedovo, G. Pirlo, "A New Methodology for the Measurement of Local Stability in Dynamical Signatures", Proc. IWFHR-4, Taipei, Taiwan, PP. 135-144, Dec. 1994.

[168] E. Argones-Rúa, D.P. López, and J.L. Alba-Castro, "Ergodic HMM-UBM System for On-Line Signature Verification", in Proc. COST 2101/2102 Conference, PP.340-347, 2009.

[169] S. Fauziyah, B. Mardiana, M. Zahariah, H. Hazura, "Signature Verification System using Support Vector Machine", MASAUM Journal of Basic and Applied Sciences, Vol.1, No. 2, September 2009.

[170] A. Mendaza-Ormaza, O. Miguel-Hurtado, I. Rubio-Polo, and R. Alonso-Moreno, "On-line Signature Biometrics using Support Vector Machine", in Proc. BIOSIG, PP.185-188, 2009.

[171] J.C.M. Romo, F.J.L. Rosas, and M. Mora-González, "On-Line Signature Verification Based on Genetic Optimization and NeuralNetwork-Driven Fuzzy Reasoning", in Proc. MICAI, PP.246-257, 2009.

[172] J. Martínez-R. and R. Alcántara-S., "On-line signature verification based on optimal feature representation and neural-networkdriven fuzzy reasoning", Proc. ICAll, July 2003.

[173] A. McCabe, J. Trevathan and W. Read, "Neural Network-based Handwritten Signature Verification", JOURNAL OF COMPUTERS, VOL. 3, NO. 8, AUGUST 2008.

[174] A. Czajka and A. Pacut, "Neural networks for signature classification and identity verification", SECURE 2002 Materiały Konferencyjne, 6-7 listopada 2002, Warszawa, tom 1, str. 1-7, 2002.

[175] J.M. Pascual-Gaspar and V. Carde noso-Payo, "Automatic Online Signature Verification Using HMMs with User-Dependent Structure", ICB 2007, LNCS 4642, PP. 1057-1066 , 2007.

[176] M. Parizeau and R. Plamondon, "a comparative analysis of regional correlation, dynamic time warping and skeletal tree matching for signature verification", IEEE Transactions on pattern analysis and machine intelligence, Vol. 12, No. 7, PP. 710-717, July 1990.

[177] C. Gruber, T. Gruber, S. Krinninger and B. Sick, "online signature verification with support vector machines based on LCSS kernel functions", IEEE trans. on systems, man and cybernetics, Vol.40, No.4, PP.1088-1100, 2010.

[178] C. Gruber, T. Gruber, and B. Sick, "Online Signature Verification with New Time Series Kernels for Support Vector Machines", ICB 2006, LNCS 3832, PP. 500-508, 2005.

[179] B. Kar and P. K. Dutta, "SVM based signature verification by fusing global and functional features", International Journal of Computer Applications (0975 - 8887), Vol. 60, No.16, PP. 34-39, December 2012.

[180] D. Muramatsu, K. Yasuda, S. Shirato and T. Matsumoto, "Camera-Based Online Signature Verification with Sequential Marginal Likelihood Change Detector", CAIP 2009, LNCS 5702, PP. 229-236, 2009.

[181] K. Yasuda, D. Muramatsu, S. Shirato and T. Matsumoto, "visual-based online signature verification using features extracted from video", Journal of Network and Computer Applications, Vol. 33, PP. 333-341, 2010.

[182] Y. Lee, "developing a biometric authentication system using dynamic signature verification statistical learning and soft computing approaches", PHD thesis, Capella University, Nov. 2004.

[183] I.Nakanishi, N. Nishiguchi, Y. Itoh and Y. Fukui, "on-line signature verification based on subband decomposition by DWT and adaptive signal processing", Electronics and Communications in Japan, Part 3, Vol. 88, No. 6, 2005.

[184] S. Koike, I. Nakanishi and Y. Itoh, "score level fusion in DWT domain on-line signature verification", the 23rd ITC-CSCC 2008, PP. 669-672, 2008.

[185] I. Nakanishi, H. Sakamoto, Y. Itoh and Y. Fukui, "Optimal User Weighting Fusion in DWT Domain On-Line Signature Verification", AVBPA 2005, LNCS 3546, PP. 758-766, 2005.

[186] L. Mi, "research on the rejection capabilities of the signature-pressure-based individual recognition system for counterfeit signatures using optimized neuro-template with gaussian function", PHD thesis, Department of engineering, Kochi University of Technology, Kochi, Japan, March 2007.

[187] L. L.-Sulem, S. Salicetti, J. Dittmann, J. Ortega-Garcia,N. Pavesic, G. Gluhchev, S. Ribaric and B. Sankur, "jointly executed research carried out on the signature modality", Biosecure Project report, July 2006.

[188] E. Maiorana, "Biometric cryptosystem using function based on-line signature recognition", Expert Systems with Applications, Vol. 37, PP. 3454-3461, 2010. 
[189] M. R. Freire, "Biometric Template Protection in Dynamic Signature Verification", Master thesis, Madrid, Nov. 2008

[190] M. R. Freire, J. Fierrez and J. Ortega-Garcia, "dynamic signature verification with template protection using helper data", ICASSP 2008.

[191] E. Maiorana, P. Campisi , and A. Neri, "Template Protection for On-line Signature-based Recognition Systems", Biometrics and Identity Management, First European Workshop, BIOID 2008, Denmark, LNCS 5372, May 2008.

[192] W. Bin and L. Z.-Chen, "On-Line Signature Verification With Two-Stage Statistical Models", Proc. 8th International Conference on Date of Conference Document Analysis and Recognition, Vol. 1, PP. 282-286, Sept. 2005.

[193] L. Nanni, A. Lumini, "Advanced methods for two-class problem formulation for on-line signature verification", Neurocomputing Vol. 69, PP. 854-857, 2006.

[194] B. LI, D. Zhang and K. Wang, "online signature verification by combining shape contexts and local features", International Journal of Image and Graphics, Vol. 6, No. 3, PP.407-420, 2006.

[195] J. P.-Leszczynska1 and A. Pacut, "Signature Verification Based on a Global Classifier That Uses Universal Forgery Features", ICCCI 2011, Part II, LNCS 6923, PP. 170-179, 2011.

[196] J. Zheng, X. Gao, E. Zhan and Z. Huang, "Algorithm of On-Line Handwriting Signature Verification Based on Discrete Fréchet Distance", ISICA 2008, LNCS 5370, PP. 461-469, 2008.

[197] M. M. Diaz, "dynamic signature verification for portable devices", Master thesis, Univ. autonoma de madrid, 2008.

[198] J. Richiardi, A. Drygajlo and L. Todesco, Promoting Diversity in Gaussian Mixture Ensembles An Application to Signature Verification, BIOID 2008, LNCS 5372, PP. 140-149, 2008.

[199] H. D. Crane and J. S. Ostrem, "Automatic signature veri?cation system using a three-axis force-sensitive pen," IEEE Trans. Syst., Man, Cybern., Vol. SMC-13, No. 3, PP. 329-337, May/Jun. 1983.

[200] J Fierrez, J Ortega-Garcia, "On-Line Signature Verification", Handbook of Biometrics, Springer, 2008.

[201] V. Mottl, M. Lange, V. Sulimova, and A. Yermakov, "Signature verification based on fusion of on-line and off-line kernels", in Proc. ICPR, PP.1-4, 2008.

[202] A. Tatarchuk, V. Sulimova, D. Windridge, V. Mottl and M. Lange, "supervised selective combining pattern recognition modalities and its application to signature verification by fusing on-line and off-line kernels", MCS 2009, LNCS 5519, PP. 324-334, 2009.

[203] F. Alonso-Fernandez, F. Fiérrez, M. Martinez-Diaz, and J. Ortega-Garcia, "Fusion of static image and dynamic information for signature verification", in Proc. ICIP, PP.2725-2728, 2009.

[204] K. K. GURRALA, "online signature verification techniques", Master Thesis, Department of Electronics and Communication Engineering, National Institute Of Technology Rourkela, 2011.

[205] C. V.-Pascual, M. F.-Zanuy and J. M. Pascual, "an efficient low cost approach for on-line signature recognition based on length normalization and fractional distances", Pattern Recognition, Vol. 42, PP. 183-193, 2009.

[206] H. Lei and V. Govindaraju, "A Study on the Consistency of Features for On-Line Signature Verification", SSPR\&SPR 2004, LNCS 3138, PP. 444-451, 2004.

[207] H. Jiao, D. Wang and Y. Zhang, "different role of friction and normal force for force-based signature verification", Proc. of the 2nd International Congress on Image and Signal Processing. Tianjin, China: IEEE, PP. 1-5, 2009.

[208] D. Ahmedt-Aristizabal, E. Delgado-Trejos, J.F.V. Bonilla, and J.A. Jaramillo-Garzón, "Dynamic signature for a closed-set identification based on nonlinear analysis", in Proc. IJCB, PP.1-8, 2011.

[209] J. C. M. ROMO and R. A. SILVA, "optimal prototype functions of features for online signature verification", International Journal of Pattern Recognition and Artificial Intelligence, Vol. 18, No. 7, 2004.

[210] D.S. Guru and H.N. Prakash, "online signature verification and recognition an approach based on symbolic representation", IEEE transactions on pattern analysis and machine intelligence, VOL. 31, NO. 6, JUNE 2009.

[211] M. F.-Santos, J. F.-Aguilar, J. O.-Garcia, "Cryptographic key generation using handwritten signature", Biometric technologies for human identification III, proceedings of SPIE, Vol. 6202, 2006.

[212] J. M. Pascual-Gaspar, M. Faundez-Zanuy, C. Vivaracho, "Fast on-line signature recognition based on VQ with time modeling", Engineering Applications of Artificial Intelligence, Vol. 24, PP. 368-377, 2011.

[213] V. Sulimova, V. Mottl and A. Tatarchuk, "multi-kernel approach to on-line signature verification", Proc. of the 8th IASTED International Conference on Signal and Image Processing, PP. 448-453, 2006.

[214] K.R.Radhika, M.K.Venkatesha and G.N. Sekhar, "On-line Signature Authentication", IJCSNS International Journal of Computer Science and Network Security, VOL.10, No.2, February 2010.

[215] A. Alizadeh, T. Alizadeh and Z. Daei, "Optimal Threshold Selection for Online Verification of Signature", Proceeding of the international multiconference of engineers and computer scientists (IMECS), 2010.

[216] F. A-Fernandez, J. F-Aguilar, and J. O-Garcia, "Sensor interoperability and fusion in signature verification: A case study using tablet PC", LNCS 3781, Proc. IWBRS, Beijing, China Springer-Verlag, PP. 180-187, Oct. 2005.

[217] S. Schimke, C. Vielhauer and J. Dittmann, "Using Adapted Levenshtein Distance for On-Line Signature Authentication", Proceedings of the 17th International Conference on Pattern Recognition (ICPR'04), 2004.

[218] J. F-Aguilar, J. O-Garcia, and J. G-Rodriguez, "target dependent score normalization techniques and their application to signature 
Council for Innovative Research www.cirworld.com
International Journal of Computers \& Technology Volume 4 No. 2, March-April, 2013, ISSN 2277-3061

verification", IEEE Trans. on systems, man, and cybernetics, Vol. 35, No. 3, 2005.

[219] S. Sayeed, N. S. Kamel and R. Besar, "Dynamic Signature Verification", College Science in India, 2007.

[220] R. S. Corradin, "Signature verification in consignment notes", Master thesis, VU University, Amsterdam,2008.

[221] J. W. Kim, H. G. Cho and E. Y. Cha, "A Study on Enhanced Dynamic Signature Verification for the Embedded System", BVAI 2005, LNCS 3704, PP. 436-446, 2005.

[222] J.-W. Kim, "An Enhanced Dynamic Signature Verification System for the Latest Smart-Phones", MulGraB 2011, CCIS 263, 2011.

[223] J. F. Aguilar, "adapted fusion schemes for multimodal biometric authentication", Madrid, may 2006.

[224] H. Lv and W. Wang, "On-Line Signature Verification Based on Dynamic Bayesian Network", ICNC 2006, Part I, LNCS 4221, PP. 507-510, 2006.

[225] J. Fierrez, J. Ortega-Garcia, "Function-Based Online Signature Verification", Chapter 12 - Part II Algorithms in Advances in Biometrics: Sensors, Algorithms and Systems, PP. 225-246, Springer, 2008.

[226] B. Li, D. Zhang and K. Wang, "Online signature verification based on null component analysis and principal component analysis", Pattern Anal. Appl. Vol. 8, No. 4, PP.345-356, 2006.

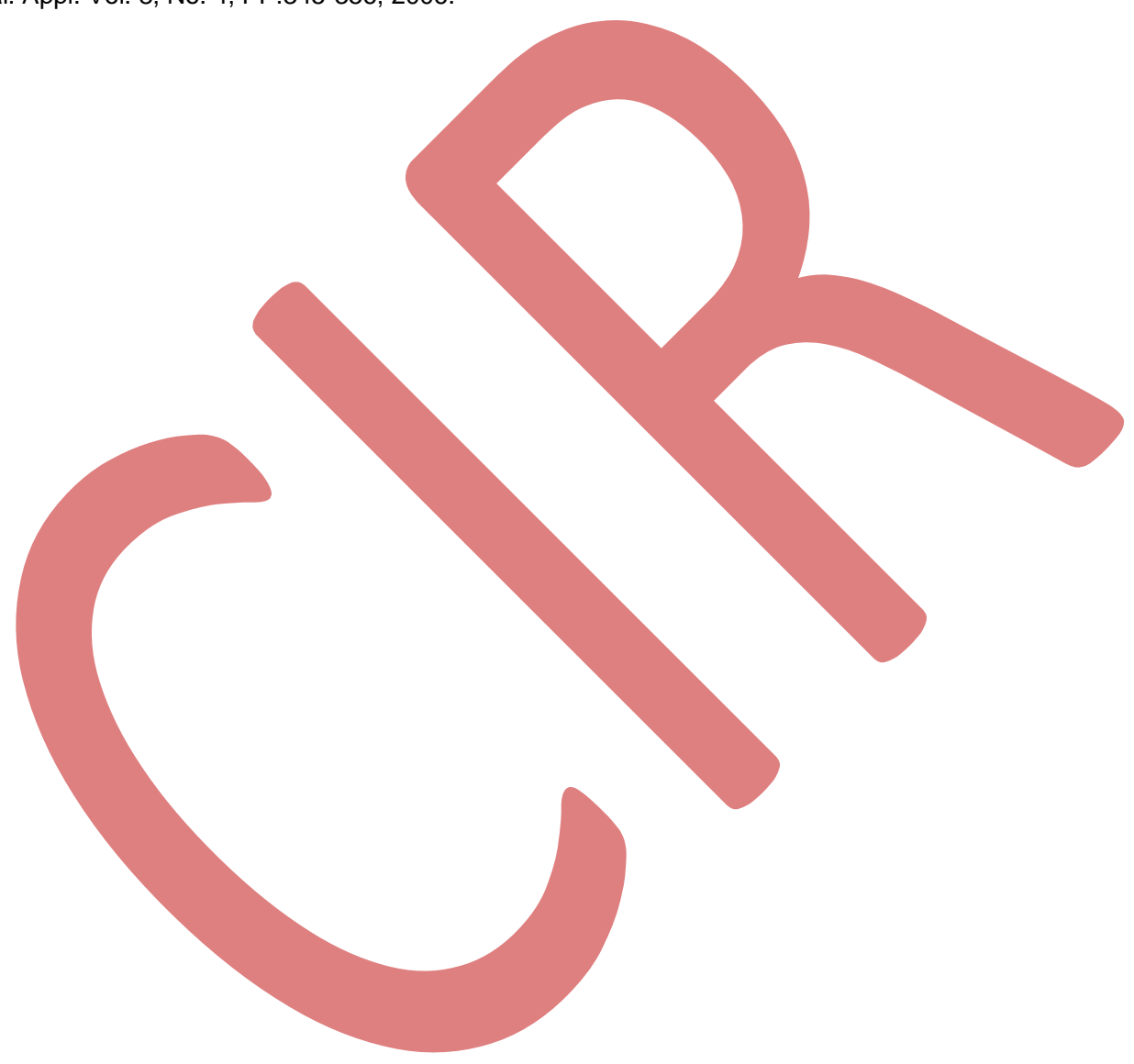

